\title{
Comparison of Oxidative Powers of DADP and TATP - A DFT Treatise
}

\section{Lemi Türker}

Department of Chemistry, Middle East Technical University, Üniversiteler, Eskişehir Yolu No: 1, 06800 Çankaya/Ankara, Turkey; e-mail: 1turker@gmail.com; lturker@metu.edu.tr

\begin{abstract}
Diacetone diperoxide (DADP) and triacetone triperoxide (TATP) are very sensitive organic-peroxide type explosives which are easily synthesized. The present density functional treatment considers oxidative powers of DADP and TATP molecules based on comparison of their interactions with certain group II-metals at the level of B3LYP/6$311++\mathrm{G}(\mathrm{d}, \mathrm{p})$. Composite systems of DADP and TATP with $\mathrm{Be}, \mathrm{Mg}$ and $\mathrm{Ca}$ have been considered. Although, in the case of beryllium composites, DADP and TATP and in the magnesium composite of DADP the organic component remains intact, Ca composites of DADP and TATP undergo ring rapture. Certain structural, electronic, quantum chemical and some spectral properties of the composites have been obtained and discussed.
\end{abstract}

\section{Introduction}

Generally, organic peroxides are a relatively unexplored class of substances. The most prominent example is acetone peroxide which was synthesized for the first time by the German chemist Richard Wolffenstein in 1895 [1]. It was found that the main fraction is a trimeric molecule (triacetone triperoxide, TATP, 3,3,6,6,9,9-hexamethyl-1,2,4,5,7,8hexoxonane). The trimer is accompanied by a dimer, known as DADP, diacetone diperoxide [2]. It has been found that the ratio of the two possible products are formed depends on the reaction conditions (mainly the concentration of the acid used) employed during the reaction. Generally, concentrated and strong acids favor the formation of DADP whereas lower acid concentrations or acid free conditions lead to the formation of TATP. It has been reported that in the presence of catalysis of tin (IV), a tetrameric form

Received: November 10, 2021; Accepted: December 2, 2021

Keywords and phrases: DADP; diacetone diperoxide; TATP; triacetone triperoxide; peroxide explosives; explosives.

Copyright (C) 2022 Lemi Türker. This is an open access article distributed under the Creative Commons Attribution License (http://creativecommons.org/licenses/by/4.0/), which permits unrestricted use, distribution, and reproduction in any medium, provided the original work is properly cited. 
can be isolated [3]. Triacetone triperoxide (TATP), diacetone diperoxide (DADP) and hexamethylene triperoxide diamine (HMTD) are sensitive and powerful organic peroxide explosives [4].

Some methods to degrade peroxide explosives (DADP, TATP, and HMTD) chemically, at room temperature have been described. Investigations indicated that a number of mixtures containing metals (e.g., zinc, copper) and metal salts (e.g., zinc sulfate, copper chloride) were effective, so that some capable of destroying TATP solutions in a few hours. Strong acids proved useful against solid peroxide materials [5].

Diacetone diperoxide (DADP) is the thermodynamically-favored one, as opposed to the more commonly formed and kinetically-favored triacetone triperoxide (TATP). Research by Matyáš et al. has shown friction sensitivity differences between DADP, HMTD and TATP [6]. Another research has indicated that conversion of TATP to DADP can occur spontaneously if conditions are favorable [7]. It is to be noted that vapor pressures of both acetone peroxides are significant to be detected by the human olfactory sense [8].

On the other hand, pathway(s) through which acetone and hydrogen peroxide follow to form TATP is/are still debatable whether DADP forms directly, or TATP forms first followed by rearrangement to DADP in solution [9]. The synthesis of TATP occurs slowly over time when hydrogen peroxide (HP) and acetone are combined for a sufficiently long period, whereas the addition of an acid catalyst accelerates the process significantly $[1,10]$. Although, addition of low to moderate levels of acid catalyst, results in a mixture of TATP and DADP, high levels of added acid forms DADP as the sole product. Some recent studies have indicated interesting behavior of DADP when cocrystallized with other explosive materials [11]. Bowden et al, have managed to characterize diacetone diperoxide [12]. On the other hand, Kahnooji et al., using density functional theory calculations have investigated relationships between the structures and performance of a series of highly energetic diacetone diperoxides [13]. Recently TATP and some of its composites have been the focus of a computational study [14].

\section{Method of Calculation}

All the structures were subjected to MM2 initial geometry optimizations leading to energy minima followed by semi-empirical PM3 self-consistent fields molecular orbital (SCF MO) method [15,16] at the restricted level [17]. Then, the structure optimizations have been achieved within the framework of Hartree-Fock (HF) and finally by using 
density functional theory (DFT) at the level of B3LYP/6-311++G(d,p) $[18,19]$. The exchange term of B3LYP consists of hybrid Hartree-Fock and local spin density (LSD) exchange functions with Becke's gradient correlation to LSD exchange [20]. It is worth mentioning that the correlation term of B3LYP consists of the Vosko, Wilk, Nusair (VWN3) local correlation functional [21] and Lee, Yang, Parr (LYP) correlation correction functional [22]. The normal mode analysis for each structure presently considered yielded no imaginary frequencies for the $3 \mathrm{~N}-6$ vibrational degrees of freedom. Note that $N$ is the number of atoms in the system and the present normal mode analysis indicates that the structure of each molecule corresponds to at least a local minimum on the potential energy surface. Furthermore, all the bond lengths were thoroughly searched in order to find out whether any bond cleavage occurred or not during the geometry optimization process. All these computations were performed by using SPARTAN 06 [23].

\section{Results and Discussion}

Since DADP and TATP molecules possess peroxide linkages their oxidative character is dominating in their reactions with other agents. However, it would be scientific curiosity to scale up their oxidative powers at least qualitatively. Their calculated electron affinity and hardness values are 377.735, 334.72 (electron affinities) and 335.795, 313.84 (hardnesses), respectively. The above mentioned properties are defined as [24],

$$
\begin{gathered}
\text { Electronegativity }=-(\mathrm{HOMO}+\mathrm{LUMO}) / 2 \\
\text { Hardness }=-(\mathrm{HOMO}-\mathrm{LUMO}) / 2
\end{gathered}
$$

See below for their HOMO and LUMO energies. The chemical hardnesses of Be, $\mathrm{Mg}$ and $\mathrm{Ca}$ are 4.5, 3.9 and 4.0, respectively [24]. Note that hard-hard or soft-soft interactions are more favored than the mixed types [25].

Figure 1 shows the optimized structures, direction of dipole moment vectors and electrostatic charges on DADP and TATP molecules. As seen in the figure the charges on oxygen atoms in DADP are almost equal to each other whereas this is not the case in TATP, especially in one of the peroxide bonds, charges on the oxygens are highly different. This should be the consequences of its conformational form which is highly puckered relative to the DADP case which is relatively a stiff molecule. 


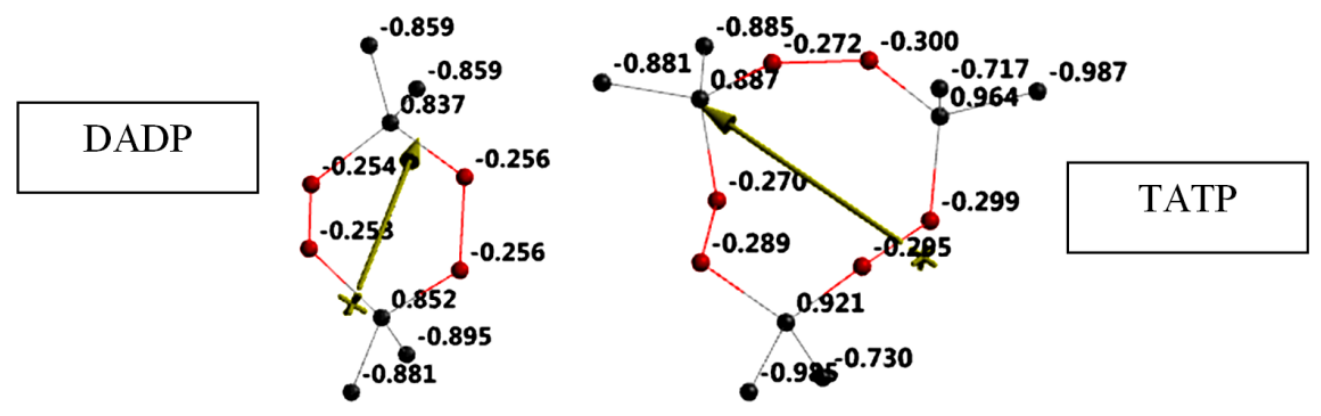

Figure 1. Optimized structures and electrostatic charges of DADP and TATP molecules (Hydrogens omitted).

Figure 2 displays the optimized structures as well as direction of the dipole moment vectors of DADP composites of beryllium, magnesium and calcium and the electrostatic charges on atoms of those composites. As seen in the figure the metals, $\mathrm{Be}, \mathrm{Mg}$ and $\mathrm{Ca}$ all possess positive charges in the order of $\mathrm{Be}<\mathrm{Mg}<\mathrm{Ca}$. It is worth mentioning that the calcium composite has a decomposed DADP structure and the charge of calcium is greater that unity in contrast to $\mathrm{Be}$ and $\mathrm{Mg}$ cases which have charges of less than unity.
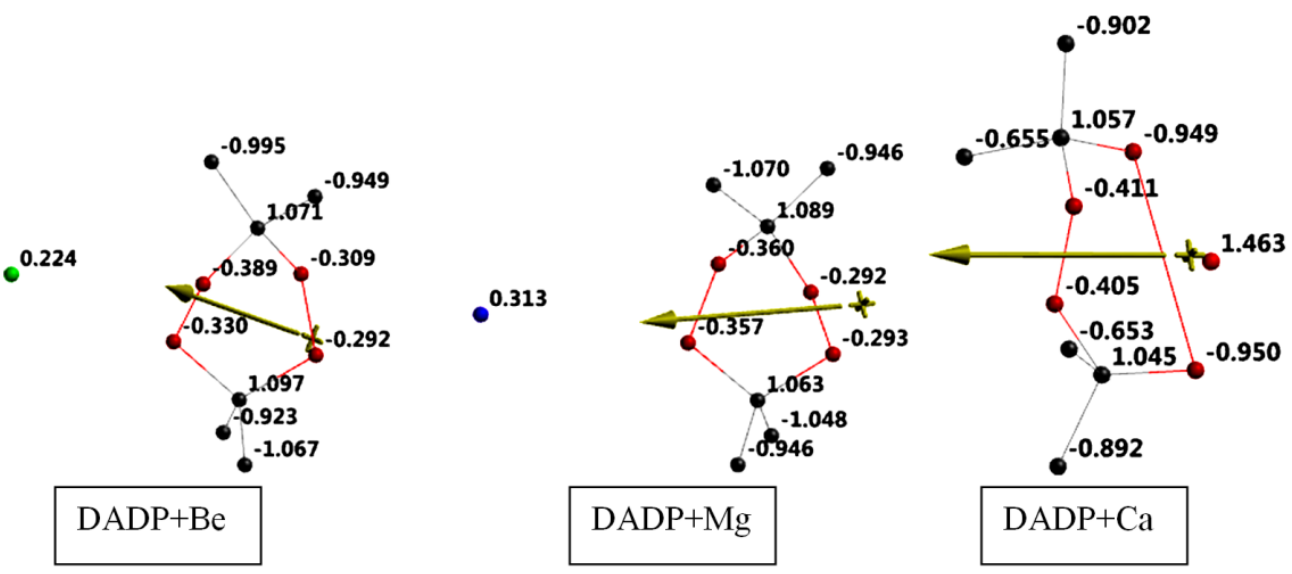

Figure 2. Optimized structures and electrostatic charges of DADP composites considered (Hydrogens omitted).

Figure 3 shows the optimized structures, direction of the dipole moment vectors and the electrostatic charges of TATP composites considered. Note that this time both the magnesium and calcium composites have the decomposed TATP structure in contrast to $\mathrm{DADP}+\mathrm{Mg}$ case. As seen in Figure 3 the order of positive charge on the metals employed is $\mathrm{Be}<\mathrm{Mg}<\mathrm{Ca}$. Note that the $\mathrm{Mg}$ and $\mathrm{Ca}$ metals have some charge greater than unity. 

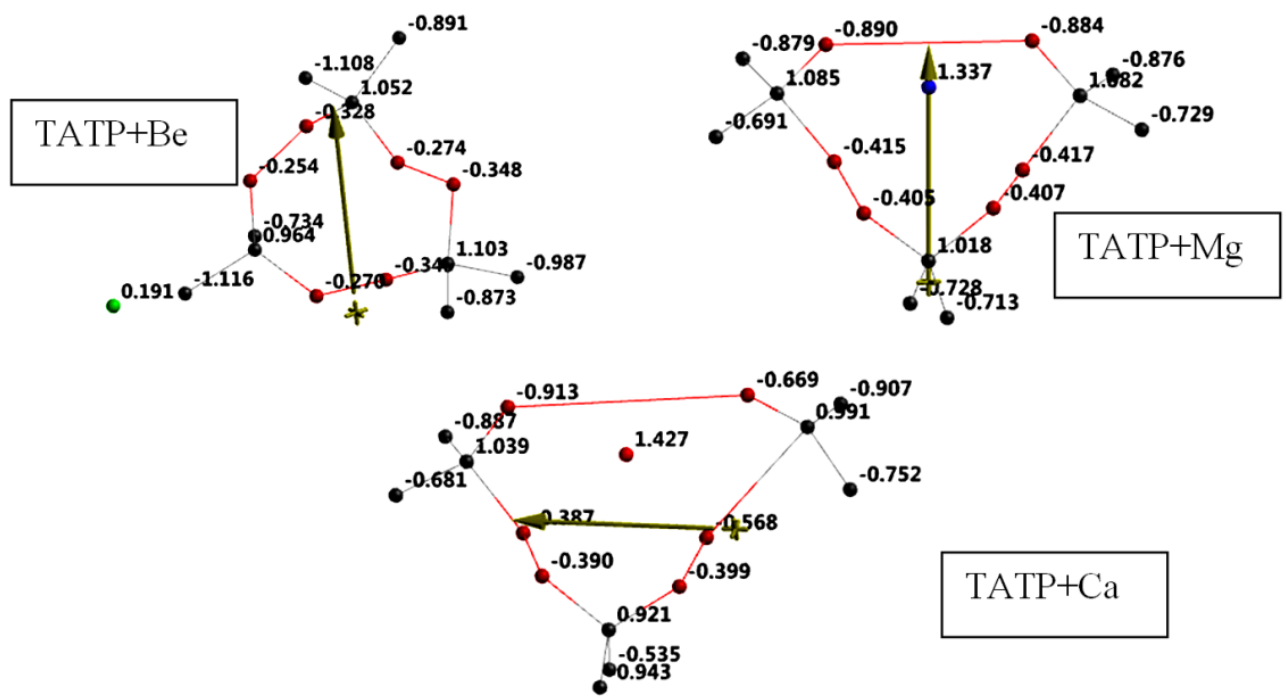

$\mathrm{TATP}+\mathrm{Ca}$

Figure 3. Optimized structures and electrostatic charges of TATP composites considered (Hydrogens omitted).

Figure 4 shows the electrostatic potential maps of DADP composites considered. Note the beryllium case in which potential field for Be remains not fused with the field of organic part whereas partially overlapped in the case of $\mathrm{Mg}$ and effectively fused in the calcium case. This fact should arise from interaction of atomic orbitals of the metals and molecular orbitals of the organic moiety accompanied by polarization effects.
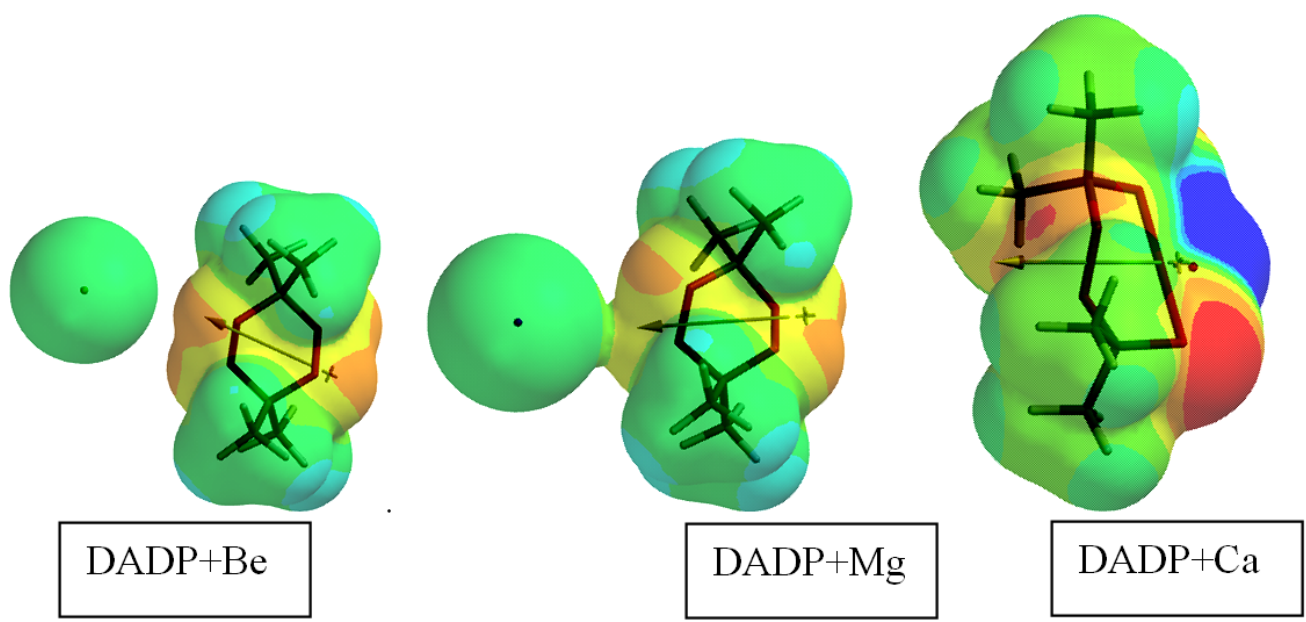

Figure 4. Electrostatic potential maps of DADP composites considered. 
The order of positive charge developed on the metals of DADP and TATP composites (see Figures 2 and 3) are $\mathrm{TATP}+\mathrm{Be}<\mathrm{DADP}+\mathrm{Be}<\mathrm{DADP}+\mathrm{Mg}<$ $\mathrm{TATP}+\mathrm{Mg}<\mathrm{DADP}+\mathrm{Ca}<\mathrm{TATP}+\mathrm{Ca}$. As indicated in the order, DADP causes more positive charge development on the beryllium atom compared to TATP but TATP is more effective than DADP on the positive charge development in the case of magnesium and calcium. Note that both the $\mathrm{Mg}$ and Ca cause decomposition of TATP structure. In other words these metals are easily oxidized by TATP acquiring themselves some positive charge. Thus, it reversely follows the ionization potentials of those metals (see Table 1) [26]. However, DADP cannot oxidize Mg although its LUMO energy is much lower than TATP (see Table 2), thus it is expected to be more effective on the oxidation of $\mathrm{Mg}$ than TATP is.

Table 1. Ionization potentials of the metals considered.

\begin{tabular}{lccc}
\hline Ionization type & $\mathrm{Be}$ & $\mathrm{Mg}$ & $\mathrm{Ca}$ \\
\hline I & 9.28 & 7.61 & 6.09 \\
II & 18.12 & 14.96 & 11.82 \\
Total & 27.42 & 22.58 & 17.91 \\
\hline
\end{tabular}

In eV. Excerpted from ref. [26].

Table 2. The HOMO, LUMO energies and $\Delta \varepsilon$ values of the structures considered.

\begin{tabular}{lccc}
\hline Structure & HOMO & LUMO & $\boldsymbol{\Delta} \boldsymbol{\varepsilon}$ \\
\hline DADP & -713.53 & -41.94 & 671.59 \\
TATP & -648.56 & -20.88 & 627.68 \\
DADP+Be & -591.76 & -124.63 & 467.13 \\
TATP+Be & -608.56 & -136.93 & 471.63 \\
DADP+Mg & -481.48 & -76.79 & 404.69 \\
TATP+Mg & -658.12 & -87.46 & 570.66 \\
DADP+Ca & -612.12 & -160.76 & 451.36 \\
TATP $+\mathrm{Ca}$ & -576.47 & -164.29 & 412.18 \\
\hline
\end{tabular}

Energies in $\mathrm{kJ} / \mathrm{mol} . \Delta \varepsilon=\varepsilon_{\mathrm{LUMO}}-\varepsilon_{\mathrm{HOMO}}$. 
Figure 5 shows the electrostatic potential maps of TATP composites considered. In electrostatic potential maps positive and negative potential regions are blue/bluish and $\mathrm{red} /$ reddish in color, respectively.
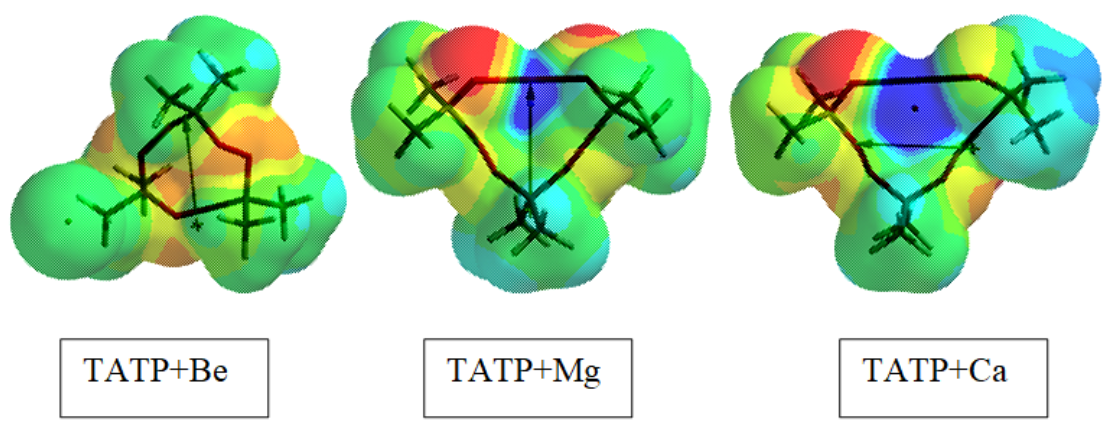

$\mathrm{TATP}+\mathrm{Ca}$

Figure 5. Electrostatic potential maps of TATP composites considered.

Figure 6 shows some molecular orbital energy levels of DADP and TATP. Some of the molecular orbital energy levels of the DADP and TATP composites ( $\mathrm{Mg}$ and $\mathrm{Ca}$ ) are shown in Figure 7.

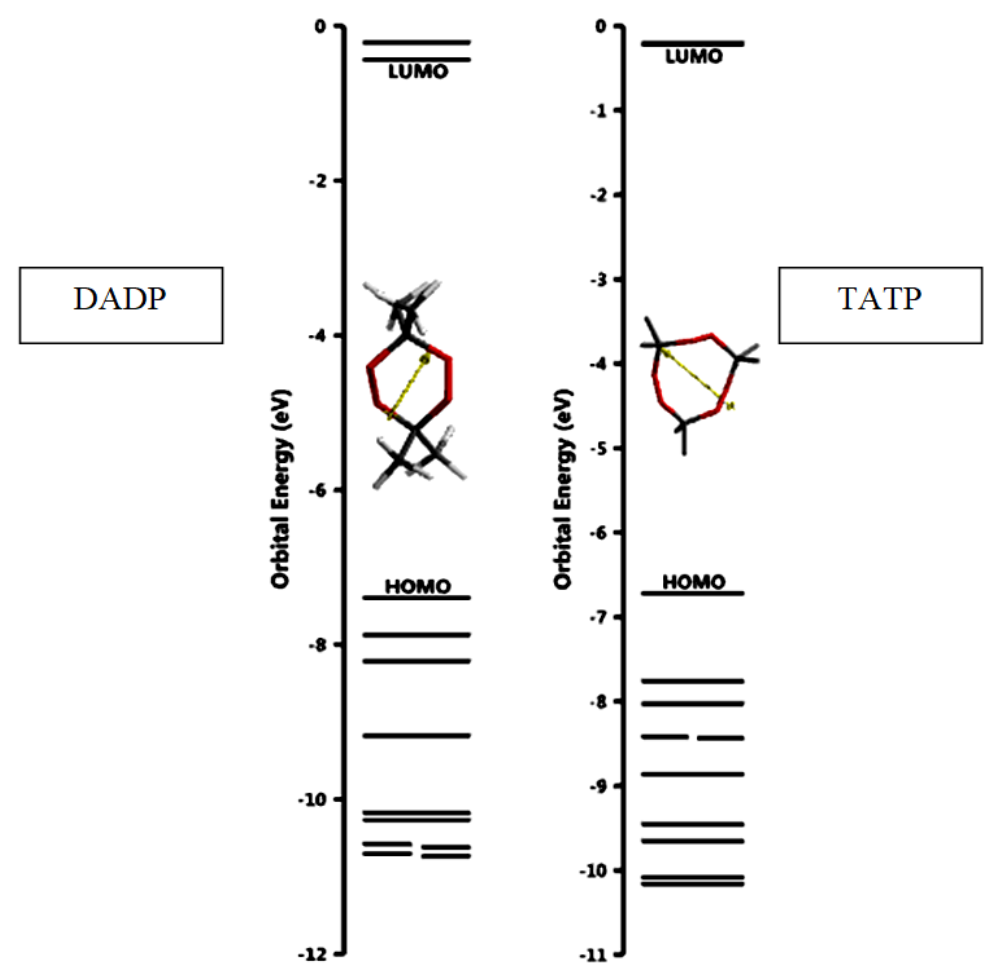

Figure 6. Some molecular orbital energy levels of DADP and TATP. 
The HOMO, LUMO energies and the interfrontier molecular orbital energy gap values $(\Delta \varepsilon)$ of the systems of interest are tabulated in Table 2. The HOMO order is $\mathrm{DADP}<\mathrm{TATP}+\mathrm{Mg}<\mathrm{TATP}<\mathrm{DADP}+\mathrm{Ca}<\mathrm{TATP}+\mathrm{Be}<\mathrm{DADP}+\mathrm{Be}<\mathrm{TATP}+\mathrm{Ca}<$ $\mathrm{DADP}+\mathrm{Mg}$, The LUMO order is TATP $+\mathrm{Ca}<\mathrm{DADP}+\mathrm{Ca}<\mathrm{TATP}+\mathrm{Be}<\mathrm{DADP}+\mathrm{Be}<$ $\mathrm{TATP}+\mathrm{Mg}<\mathrm{DADP}+\mathrm{Mg}<\mathrm{DADP}<\mathrm{TATP}$. As seen in the table the metals raise up the HOMO energy level as compared to the HOMO of TATP and DADP. Note that in the Ca case DADP structure decomposed. Whereas the LUMO level of DADP has been lowered by the effect of those metals. However, it is to be mentioned that $\mathrm{Mg}$ is less effective than the beryllium in lowering the LUMO level. In general electron donating substituents raise the frontier molecular orbitals (HOMO and LUMO) but electron attracting ones lower [25] whereas an extending conjugation narrows the interfrontier molecular orbital energy gap $(\Delta \varepsilon)$. In the present case the metals act as if they are donating and attracting electrons at unequal extends. Namely, certain kind of donation and back donation process is occurring.
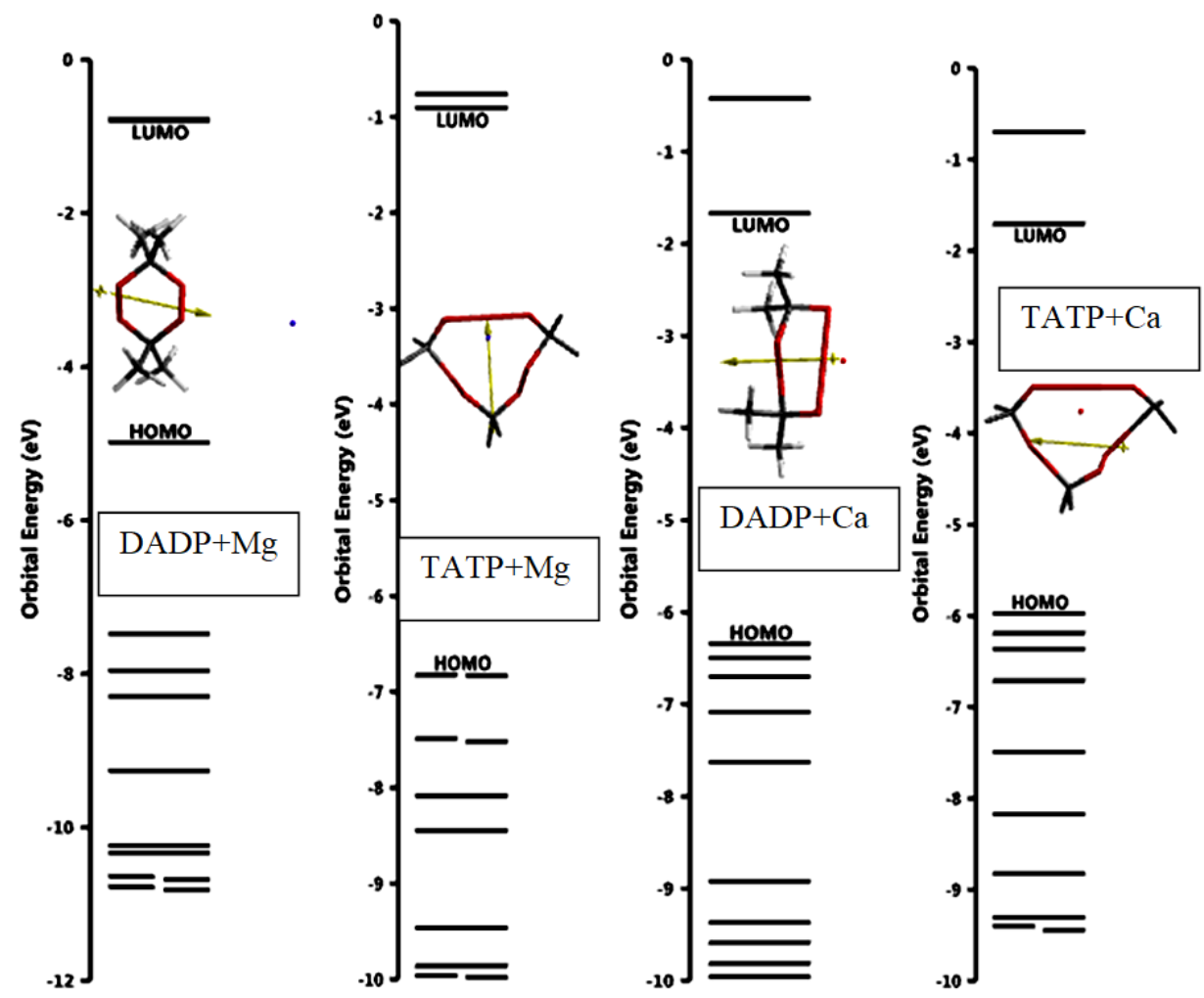

Figure 7. Some molecular orbital energy levels of $\mathrm{Mg}$ and $\mathrm{Ca}$ composites of DADP and TATP. 
The order of $\Delta \varepsilon$ values is DADP $+\mathrm{Mg}<\mathrm{TATP}+\mathrm{Ca}<\mathrm{DADP}+\mathrm{Ca}<\mathrm{DADP}+\mathrm{Be}<$ $\mathrm{TATP}+\mathrm{Be}<\mathrm{TATP}+\mathrm{Mg}<\mathrm{TATP}<\mathrm{DADP}$. Note that TATP possesses much narrower interfrontier molecular orbital energy gap $(\Delta \varepsilon)$ than DADP. On the other hand, the impact sensitivity has been reversely correlated with $\Delta \varepsilon$ value $[27,28]$. Thus TATP should be more sensitive to impact stimulus than DADP is. Figure 8 shows the LUMOs of DADP and TATP molecules.

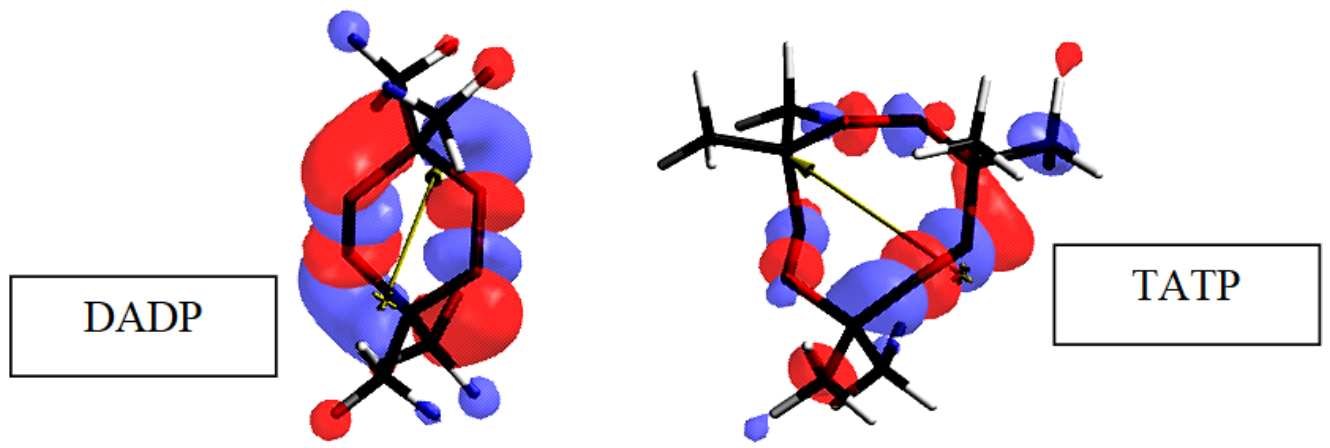

Figure 8. The LUMOs of DADP and TATP molecules.

Figure 9 shows the LUMO maps of DADP and TATP. A LUMO map displays the absolute value of the LUMO on the electron density surface. The blue color stands for the maximum value of the LUMO and the color red, the minimum value. Thus, they are correlated with the most and least susceptible sites for their potential reduced sites in a redox reaction.
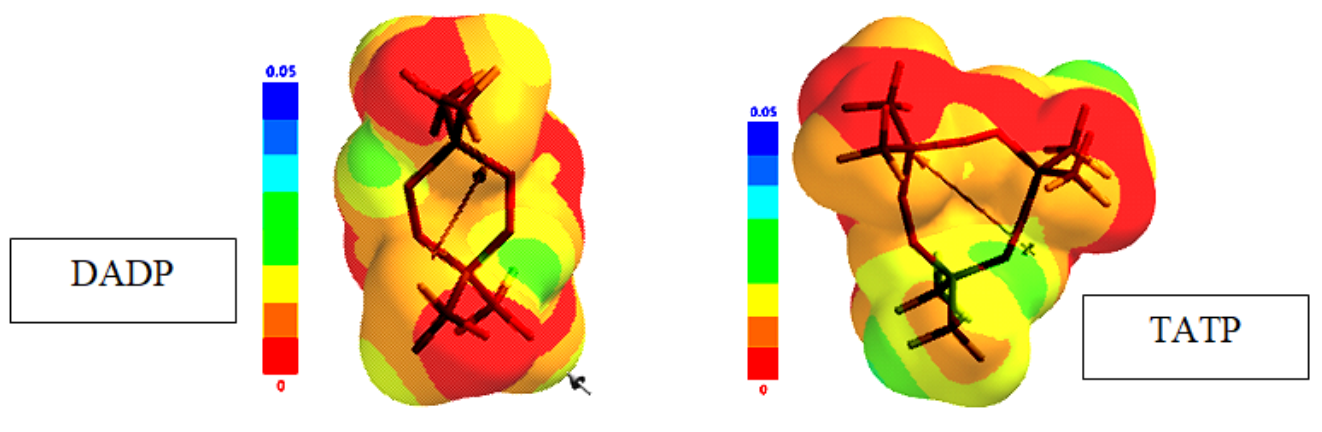

Figure 9. LUMO maps of DADP and TATP.

Figures 10-12 comparatively show the calculated (time-dependent DFT) UV-VIS spectra of composites of DADP and TATP. As seen in the figures, the beryllium 
composites of DADP and TATP are very similar to each other but magnesium composite of TATP exhibits hypsochromic effect [29] to lower wavelengths. Note that the order of $\Delta \varepsilon$ values is $\mathrm{DADP}+\mathrm{Mg}<\mathrm{TATP}+\mathrm{Ca}<\mathrm{DADP}+\mathrm{Ca}<\mathrm{DADP}+\mathrm{Be}<\mathrm{TATP}+\mathrm{Be}<$ TATP $+\mathrm{Mg}<$ TATP $<$ DADP (see Table 2). However, it is to be remembered that in $\mathrm{TATP}+\mathrm{Mg}$ composite one of the peroxide linkages has been broken. Whereas, the spectrum of TATP $+\mathrm{Ca}$, like $\mathrm{TATP}+\mathrm{Mg}$, spreads over the whole ultraviolet region. All these subtle changes in the spectra of decomposed composites should arise from special characteristics of the products of the decompositions.
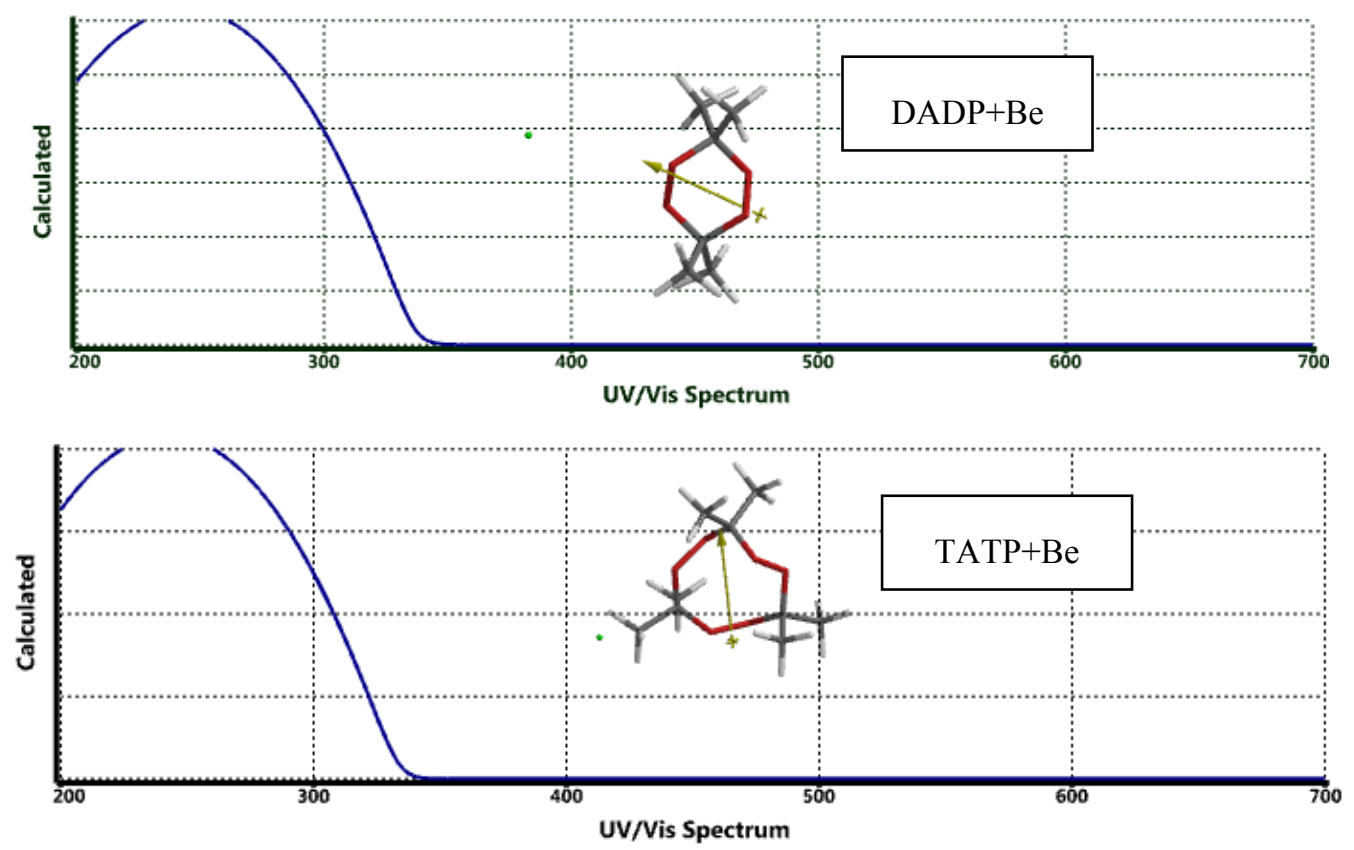

Figure 10. Calculated UV-VIS spectra of beryllium composites of DADP and TATP.

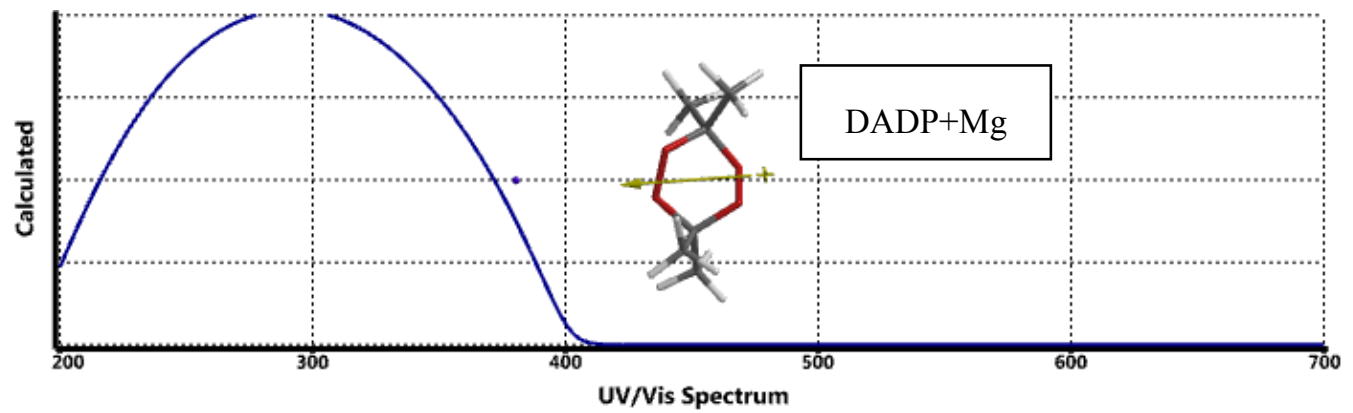




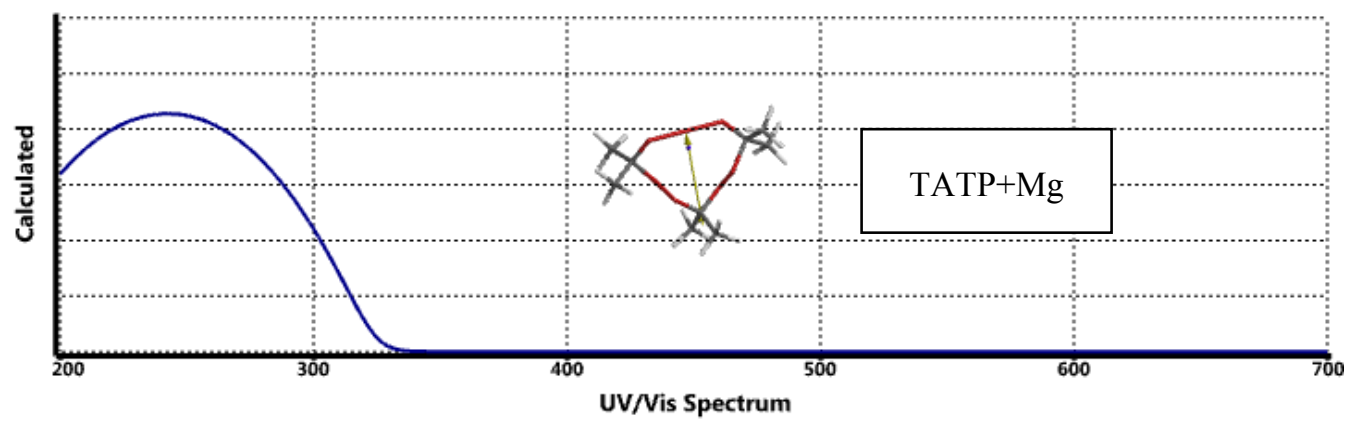

Figure 11. Calculated UV-VIS spectra of magnesium composites of DADP and TATP.
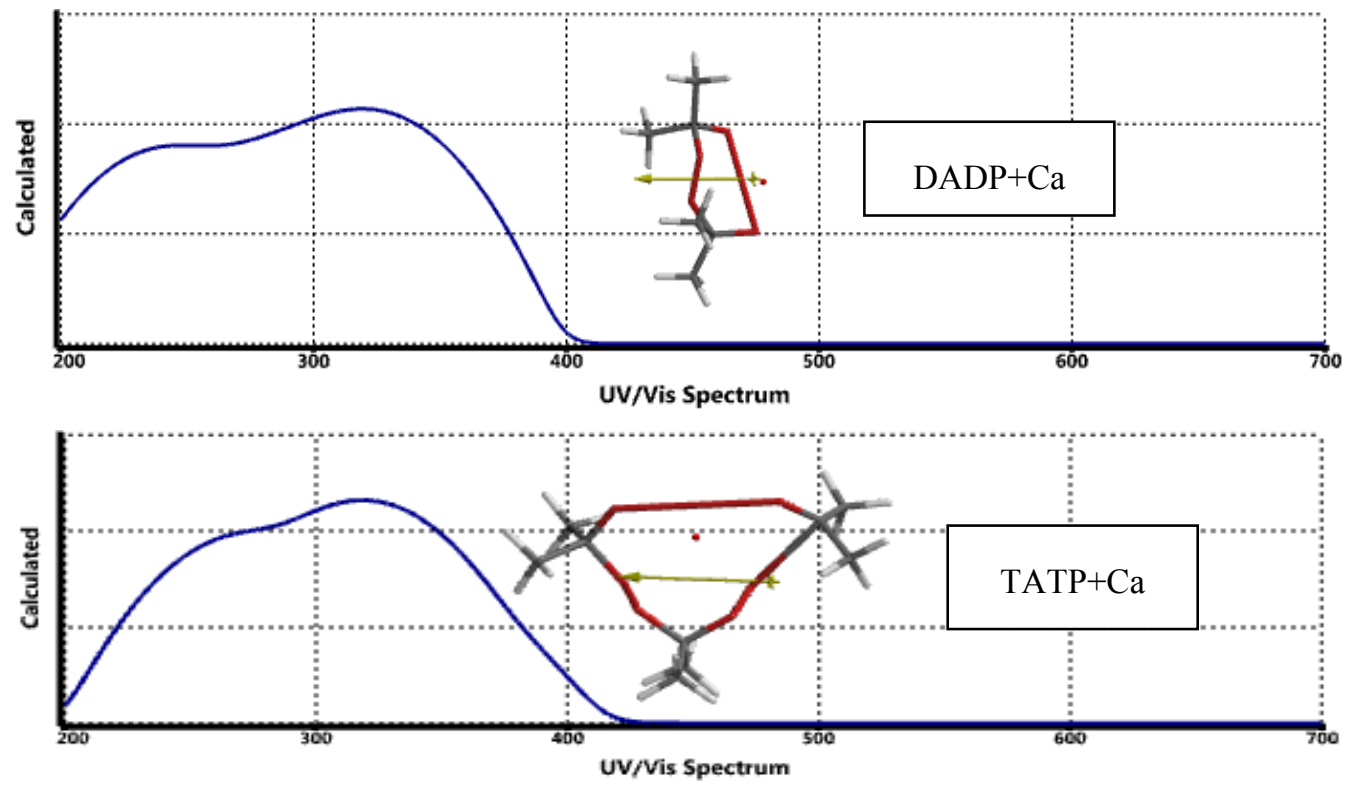

Figure 12. Calculated UV-VIS spectra of calcium composites of DADP and TATP.

\section{Conclusion}

The present density functional investigation (within the constraints of the theory and basis set applied) compares the oxidative powers of DADP and TATP via their effects on composites with some group -II metals, $\mathrm{Be}, \mathrm{Mg}$ and $\mathrm{Ca}$. Investigation has revealed that DADP component remains intact in the presence of beryllium, and magnesium whereas in the cases of $\mathrm{Mg}$ and $\mathrm{Ca}$ composites, one of the peroxide bonds of TATP is ruptured. DADP molecule also undergoes decomposition by the rupture of a peroxide bond in the presence of calcium. In TATP + Ca composite, not only the peroxide bond but also one of the $\mathrm{C}-\mathrm{O}$ bonds incident to the same carbon atom has been broken. In all the decomposed 
DADP and TATP composites metal atoms possess some positive charge greater than unity. DADP cannot oxidize magnesium. Comparing charges appeared on calcium atoms in the decomposed composites, DADP produces slightly more positive charge than TATP. These decompositions of the composites involve transfer of some electron population from the metal atom to DADP or TATP molecule.

\section{References}

[1] Wolffenstein, R. (1895). Ueber die Einwirkung von Wasserstoffsuperoxyd auf Aceton und Mesityloxyd. Ber. Dtsch. Chem. Ges., 28(2), 2265-2269. https://doi.org/10.1002/cber.189502802208

[2] Matyas, R., \& Pachman, J. (2010). Study of TATP: Influence of reaction conditions on product composition. Propellants Explos. Pyrotech., 35, 31-37. https://doi.org/10.1002/prep.200800044

[3] Jiang, H., Chu, G., Gong, H., \& Qiao, Q. (1999). Tin chloride catalyzed oxidation of acetone with hydrogen peroxide to tetrameric acetone peroxide. J. Chem. Res., 28(4), 288-289. https://doi.org/10.1039/a809955c

[4] Bali, M.S., Wallace, L., Day, A.I., \& Armitt, D. (2014). Cyclic pentanone peroxide: Sensitiveness and suitability as a model for triacetone triperoxide. Journal of Forensic Sciences, 59, 936 - 942. https://doi.org/10.1111/1556-4029.12439

[5] Oxley, J., Smith, J.L., Huang, J., \& Luo, W. (2009). Destruction of peroxide explosives. Journal of Forensic Sciences, 54(5), 1029-33. https://doi.org/10.1111/j.1556-4029.2009.01130.x

[6] Matyáš, R., Selesovsky, J., \& Musil, T. (2012 ). Sensitivity to friction for primary explosives. J. Hazard Mater., 213-214, 236-241. https://doi.org/10.1016/j.jhazmat.2012.01.085

[7] Matyáš, R., Pachman, J., \& Ang, H.-G. (2009). Study of TATP: Spontaneous transformation of TATP to DADP. Propellants Explos. Pyrotech., 34(6), 484-488. https://doi.org/10.1002/prep.200800043

[8] Oxley, C., Smith, J.L., Luo, W., \& Brady, J. (2009). Determining the vapor pressures of diacetone diperoxide (DADP) and hexamethylene triperoxide diamine (HMTD). Propellants Explos. Pyrotech., 34(6), 539-543. https://doi.org/10.1002/prep.200800073

[9] Oxley, J.C., Smith, J.L., Steinkamp, L., \& Zhang, G. (2013). Factors influencing triacetone triperoxide (TATP) and diacetone diperoxide (DADP) formation: Part 2. Propellants Explos. Pyrotech., 38(6), 841-851. https://doi.org/10.1002/prep.201200215 
[10] Oxley, J.C., Smith, J.L., Bowden, P.R., \& Rettinger, R.C. (2013). Factors influencing triacetone triperoxide (TATP) and diacetone diperoxide (DADP) Formation: Part I. Propellants Explos. Pyrotech., 38(2), 244 -254. https://doi.org/10.1002/prep.201200116

[11] Landenberger, K.B., Bolton. O., \& Matzger, A.J. (2015). Energetic-energetic cocrystals of diacetone diperoxide (DADP): Dramatic and divergent sensitivity modifications via cocrystallization. J. Am. Chem. Soc., 137, 5074-5079.

https://doi.org/10.1021/jacs.5b00661

[12] Bowden, P.R., Tappan, B.C., Manner, V.W., Preston, D.N., \&. Scott, B.L. (2017). Characterization of diacetone diperoxide (DADP). AIP Conference Proceedings, 1793, 040010. https://doi.org/10.1063/1.4971504

[13] Kahnooji, M., Pandas, H.M., Mirzaei, M., \& Peyghan, A.A. (2015). Explosive properties of nanosized diacetone diperoxide and its nitro derivatives: a DFT study. Monatsh Chem., 146(9), 1401-1408. https://doi.org/10.1007/s00706-015-1419-6

[14] Türker, L. (2022). Interaction of TATP with some group II metals - A DFT treatment. Earthline Journal of Chemical Sciences, 7(1), 1-16.

https://doi.org/10.34198/ejcs.7122.116

[15] Stewart, J.J.P. (1989). Optimization of parameters for semi empirical methods I. $J$. Comput. Chem., 10, 209-220. https://doi.org/10.1002/jcc.540100208

[16] Stewart, J.J.P. (1989). Optimization of parameters for semi empirical methods II. $J$. Comput. Chem., 10, 221-264. https://doi.org/10.1002/jcc.540100209

[17] Leach, A.R. (1997). Molecular modeling. Essex: Longman.

[18] Kohn, W., \& Sham, L.J. (1965). Self-consistent equations including exchange and correlation effects. Phys. Rev., 140, 1133-1138.

https://doi.org/10.1103/PhysRev.140.A1133

[19] Parr, R.G., \& Yang, W. (1989). Density functional theory of atoms and molecules. London: Oxford University Press.

[20] Becke, A.D. (1988). Density-functional exchange-energy approximation with correct asymptotic behavior. Phys. Rev. A, 38, 3098-3100.

https://doi.org/10.1103/PhysRevA.38.3098

[21] Vosko, S.H., Vilk, L., \& Nusair, M. (1980). Accurate spin-dependent electron liquid correlation energies for local spin density calculations: a critical analysis. Can. J. Phys., 58, 1200-1211. https://doi.org/10.1139/p80-159

[22] Lee, C., Yang, W., \& Parr, R.G. (1988). Development of the Colle-Salvetti correlation energy formula into a functional of the electron density. Phys. Rev. B, 37, 785-789.

https://doi.org/10.1103/PhysRevB.37.785 
[23] SPARTAN 06 (2006). Wavefunction Inc. Irvine CA, USA.

[24] Pearson, R.G. (1997). Chemical hardness. Weinheim: Wiley-VCH. https://doi.org/10.1002/3527606173

[25] Fleming, I. (1973). Frontier orbitals and organic reactions. London: Wiley.

[26] Durrant, P. J., \& Durrant, B. (1972). Introduction to advanced inorganic chemistry. London: Longman.

[27] Anbu, V., Vijayalakshmi, K.A., Karunathan, R., Stephen, A.D., \& Nidhin, P.V. (2019). Explosives properties of high energetic trinitrophenyl nitramide molecules: A DFT and AIM analysis. Arabian Journal of Chemistry, 12(5), 621-632. https://doi.org/10.1016/j.arabjc.2016.09.023

[28] Badders, N.R., Wei, C., Aldeeb, A.A., Rogers, W.J., \& Mannan, M.S. (2006). Predicting the impact sensitivities of polynitro compounds using quantum chemical descriptors. Journal of Energetic Materials, 24, 17-33. https://doi.org/10.1080/07370650500374326

[29] Matsuoka, M. (1990). Infrared absorbing dyes. New York: Plenum Press. 